UCRL-JC-124164

PREPRINT

CONF- $9609 / 32--2$

\title{
Neural Networks and Wavelet Analysis in the Computer Interpretation of Pulse Oximetry Data
}

\author{
Farid U. Dowla, \\ Paul G. Skokowski \\ and \\ Richard R. Leach, Jr. \\ Lawrence Livermore National Laboratory
}
RECEIVED
JUN 241996
OSTI

This paper was prepared for submittal to the 1996 IEEE Workshop on Neural Networks and Signal Processing, September 4-6, Kyoto, Japan

March 1996

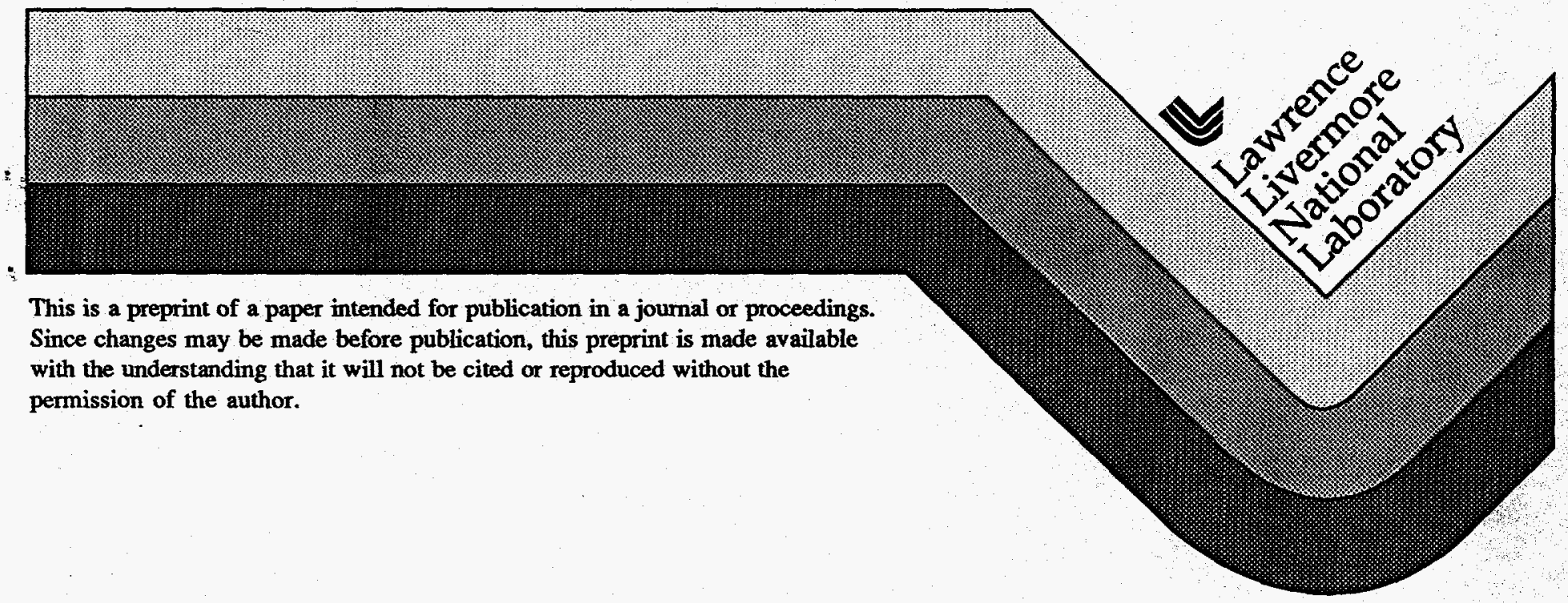




\section{DISCLAIMER}

This document was prepared as an account of work sponsored by an agency of the United States Government. Neither the United States Government nor the University of California nor any of their employees, makes any warranty, express or implied, or assumes any legal liability of responsibility for the accuracy, completeness, or usefulness of any information, apparatus, product, or process disclosed, or represents that its use would not infringe privately owned rights. Reference herein to any specific commercial products, process, or service by trade name, trademark, manufacturer, or otherwise, does not necessarily constitute or imply its endorsement, recommendation, or favoring by the United States Government or the University of California. The views and opinions of authors expressed herein do not necessarily state or reflect those of the United States Government or the University of California, and shall not be used for advertising or product endorsement purposes. 


\section{DISCLAIMER}

Portions of this document may be illegible in electronic image products. Images are produced from the best available original document. 



\title{
NEURAL NETWORKS AND WAVELET ANALYSIS IN THE COMPUTER INTERPRETATION OF PULSE OXIMETRY DATA
}

\author{
Farid U. Dowla, Paul G. Skokowski and Richard R. Leach Jr. \\ Lawrence Livermore National Laboratory \\ University of California
}

\begin{abstract}
Pulse oximeters determine the oxygen saturation level of blood by measuring the light absorption of arterial blood. The sensor consists of red and infrared light sources and photodetectors. A method based on neural networks and wavelet analysis is developed for improved saturation estimation in the presence of sensor motion. Spectral and correlation functions of the dual channel oximetry data are used by a backpropagation neural network to characterize the type of motion. Amplitude ratios of red to infrared signals as a function of time scale are obtained from the multiresolution wavelet decomposition of the two-channel data. Motion class and amplitude ratios are then combined to obtain a short-time estimate of the oxygen saturation level. A final estimate of oxygen saturation is obtained by applying a $15 \mathrm{~s}$ smoothing filter on the short-time measurements based on $3.5 \mathrm{~s}$ windows sampled every $1.75 \mathrm{~s}$. The design employs two backpropagation neural networks. The first neural network determines the motion characteristics and the second network determines the saturation estimate. Our approach utilizes waveform analysis in contrast to the standard algorithms that are based on the successful detection of peaks and troughs in the signal. The proposed algorithm is numerically efficient and has stable characteristics with a reduced false alarm rate with a small loss in detection. The method can be rapidly developed on a digital signal processing platform.
\end{abstract}

\section{INTRODUCTION}

Pulse oximeters determine the oxygen saturation level of blood by measuring light absorption of arterial blood using a sensor comprised of a dual light source and photodetectors. Since the electronic pulse oximeter was first introduced in clinical care about 15 years ago, the use of this device today has become a standard in general anesthesia. With potential applications in ambulatory health care, use of pulse oximeters is expected to increase significantly in the next few years. 
The underlying physical basis of the pulse oximeter is Beer's law which relates the intensity of light transmitted to the amount of solute concentration with the equation:

$$
I_{\text {trans }}=I_{\text {in }} \mathrm{e}^{-D \cdot C \cdot \alpha(\lambda)}
$$

where $I_{\text {trans }}$ is the intensity of transmitted light, $I_{\text {in }}$ is the intensity of incident light, $\mathrm{D}$ is the distance light is transmitted through the solute, $\mathrm{C}$ is the concentration of solute, and $\alpha(\lambda)$ is the extinction or absorption coefficient of the solute at wavelength $\lambda$.

To estimate the oxygen saturation, a two-solute concentration is assumed. With $\mathrm{c}_{\mathrm{o}}$ representing the oxygen hemoglobin, and $\mathrm{c}_{\mathrm{r}}$ representing the reduced or deoxyhemoglobin solute concentrations, a measure blood oxygen saturation level $\mathrm{S}$ can be defined:

$$
S=\frac{c_{0}}{c_{r}+c_{o}}
$$

In the literature [2], use of the symbols $\mathrm{SaO}_{2}$ and $\mathrm{SpO}_{2}$ is the common practice; however in this paper we will use $S$ to represent blood oxygen saturation level. When photodiodes are placed across the finger tips, the amount of light that passes through the tissue is different for the two different wavelengths (red and infrared). Since oxyhemoglobin absorbs more infrared light and deoxyhemoglobin absorbs more red light, the absorption of infrared light relative to the red light increases with oxyhemoglobin. The ratio of the absorption coefficients can be used to determine the oxygen saturation of blood. This measurement indicates the cardiopulmonary state of the patient.

Our goal is to estimate saturation as the function of time. By writing the extinction coefficients as $\alpha_{o}(\lambda)$ and $\alpha_{r}(\lambda)$ for the two solutes, equation (1) can be written as,

$$
I_{\text {trans }}(\lambda, t)=I_{\text {in }}(\lambda) e^{\left.j-D(t)\left[c_{0}(t) \alpha_{o}(\lambda)+c_{r}(t) \alpha_{r}(\lambda)\right]\right\}}
$$

By taking the derivative of the logarithm of equation (3) we get,

$$
x(\lambda, \mathrm{t})=-\alpha_{o}(\lambda) \hat{\mathrm{c}}_{\mathrm{o}}(t)-\alpha_{r}(\lambda) \hat{\mathrm{c}}_{\mathrm{r}}(t)
$$

where $x(\lambda, t)=\frac{\partial}{\partial t} \log \left(\mathrm{I}_{\text {trens }}(\lambda, t)\right), \quad \hat{\mathrm{c}}_{\mathrm{o}}(t)=\frac{\partial}{\partial t}\left[\mathrm{c}_{\mathrm{o}}(\mathrm{t}) \mathrm{D}(\mathrm{t})\right]$, and $\hat{c}_{r}(t)=\frac{\partial}{\partial t}\left[c_{r}(t) D(t)\right]$. Using two different wavelengths representing red and infrared lights, from equation (4) we have, 


$$
\begin{array}{lll}
g(t)=-\alpha_{o}\left(\lambda_{\text {red }}\right) & \hat{\mathrm{c}}_{\mathrm{o}}(t)-\alpha_{r}\left(\lambda_{\text {red }}\right) & \hat{\mathrm{c}}_{\mathrm{r}}(t) \\
h(t)=-\alpha_{o}\left(\lambda_{\text {ired }}\right) & \hat{\mathrm{c}}_{\mathrm{o}}(t)-\alpha_{r}\left(\lambda_{\text {ired }}\right) & \hat{\mathrm{c}}_{\mathrm{r}}(t)
\end{array}
$$

where $g(t)=x\left(\lambda_{\text {red }}, t\right)$ and $h(t)=x\left(\lambda_{\text {ird }}, t\right)$. Hence, by measuring the dual channel signals representing red and infrared lights, and by knowing the extinction parameters $\alpha^{\prime} s$, equation (5) can be used to determine $\hat{\mathrm{c}}_{\mathrm{r}}$ and $\hat{\mathrm{c}}_{\mathrm{o}}$. Assuming that the solute concentrations are constant over short time periods it can be shown that is $S=\hat{c}_{o} /\left(\hat{c}_{r}+\hat{c}_{0}\right)$. In summary, using a dual light source we can solve for $S$ even when the distance traversed $D(t)$ and the light intensities are unknown.

An approach to saturation estimation is to solve simply for the least squares amplitude ratio estimate $\eta$ between $h(t)$ and $g(t)$ as given by,

$$
\eta=\frac{\int_{1} h(t) g(t) d t}{\int h^{2}(t) d t}
$$

and then to use a calibration relationship between $\eta$ and Sto determine the later.

Sensor or patient motion can complicate the interpretation of the signals $g(t)$ and $h(t)$. The effect of patient motion is an ill-understood problem and there is a high incidence of false alarm due to poor saturation estimates triggered by patient movements. In the presence of sensor motion, pulse oximeters based on simple algorithms are unstable, particularly for use in environments such as patient rehabilitation and home care. The focus of this work is on the problem of robust estimation of arterial oxygen in the presence of sensor recording problems associated with motion.

In section 2 we discuss the problem of motion detection and develop a solution using a backpropagation network operating on the correlation and spectral data as inputs to the network. The use of wavelets for saturation estimation is discussed in section 3 . A complete system that combines motion detection and saturation estimation is discussed in section 4 . In section 5 we discuss possible areas of for further improvements that can be made over the proposed design.

\section{MOTION DETECTION USING NEURAL NETWORKS}

Motion can cause serious difficulties in the monitoring of a variety of medical signal such as the EEG, EKG, and EMG, including pulse oximetry. Signal characteristics vary not only from patient to patient, but can vary even for the same patient at different times. The backpropagation neural network is an ideal tool for the detection and classification of such complex ill-defined signals. 
A large database of training examples can be compiled using many hours of data from multiple patients. The number examples should be at least a 100 times larger than the number of weights in the network, and these examples should come from a variety of different types of saturation and recording conditions.

\subsection{Network Architecture}

As shown in Figure 1 , the second order statistics of $g(t)$ and $h(t)$ are computed and fed into a backpropagation neural network. In particular, we compute the autocorrelation and the cross-correlation functions of $h(t)$ and $g(t)$, together with the high-resolution AR spectral estimates of these two channels by segmenting the continuous data streams into short segments of $3.5 \mathrm{~s}$. The network architecture consists of one hidden layer with three input units and one output unit. In general, the number of input units depends on the number of points spectral and correlation functions. For a $3.5 \mathrm{~s}$ segment and with a sampling period of $0.0175 \mathrm{~s}$, there are 200 data points. In order to reduce the dimensions of the input vector, the correlation and spectral functions were decimated, being careful that both resolution and the general shape of the functions were preserved. We found that 50 points for are sufficient to represent these functions; i.e., there were 150 input units.

\subsection{Ranking the Motion Corrupted Data}

The training procedure is based on ranking the type of motion by visual inspection of the data; i.e., analysts ranked the quality of the data by looking at the raw data along with its spectral and correlation functions. Although a tedious process, developing an automated user-friendly environment allow analysts to go through hundreds of thousands of examples quite rapidly. We observed that the ranks obtained by visual inspection of the data are consistent when obtained with a set of pre-determined criteria such as spectral bandwidths, number of peaks, and the ratio of energy between high and low frequencies, etc. In summary, each $3.5 \mathrm{~s}$ segment data is ranked by an analyst on a scale from 0.1 (worst) to 0.9 (best) by looking at the spectral and autocorrelation functions plotted on the screen. Once a large number of such examples had been catalogued, the backpropagation network was trained. Using the ranks determined by the analysts, the backpropagation network was trained with the sampled correlation and spectral functions as inputs and the rank as the output. A detailed discussion on developing neural networks is discussed in [1].

Trained with about 50,000 examples, the backpropagation network was able to distinguish various types of motion on untrained data. We found that the motion classification problem with spectral and correlation functions as inputs, and data quality or ranks specified by an analyst is an ideal solution to ill-defined problems of this nature. The approach outlined is both easy to implement and we 
were able to address large variations in the data, from the highly distorted to the usual periodic oximetry data. We believe this approach to motion detection can also be used in other medical or non-medical data interpretation problems.

\section{SATURATION ESTIMATION USING WAVELETS}

As noted earlier from the amplitude ratio parameter $\eta$, the saturation estimate can be determined using a simple calibration procedure. Our approach was to decompose the signals $g(t)$ and $h(t)$ into their wavelet coefficients, estimate a set of amplitude ratio parameters $\eta$ 's at each of the wavelet transform scales, combine the $\eta$ 's together with the motion rankings to get a final estimate of the blood oxygen saturation estimate. The details of the wavelet decomposition method is discussed next.

Given the signals $g(t)$ and $h(t)$ from red and infrared sensors, we take the wavelet transform of the two channel data and obtain two sets of sequences:

$\left\{g_{1}(t), g_{2}(t), \cdots, g_{N}(t)\right\}$ and $\left\{h_{1}(t), h_{2}(t), \cdots, h_{N}(t)\right\}$ corresponding to a $N$-scale wavelet decomposition. Since the wavelet transform is a linear transform of the form:

$$
g_{k}(t)=\frac{1}{\sqrt{a_{k}}} \int_{\tau} g(\tau) w\left((\tau-t) / a_{k}\right) d \tau
$$

where $a_{k}$ 's are the discrete time scales and $\mathrm{w}(\mathrm{t})$ is the analyzing wavelet, it can be shown that wavelet coefficients preserve the amplitude ratio of the signals. Hence, we have $N$ estimates of the amplitude ratio parameter from $g(t)$ and $h(t)$ given by,

$$
\eta_{k}=\int_{1} g_{k}(t) h_{k}(t) d t / \int g_{k}^{2}(t) d t, \text { for } \mathrm{k}=1, \cdots, \mathrm{N}
$$

We have used both pyramid and multiresolution algorithms [6] for computing the wavelet coefficients. However, the multiresolution is the preferred algorithm in this application as its preserves the number of data points for each scale, and from a visualization and noise detection perspective the output form of the multiresolution algorithm is quite useful. The multiresolution algorithm essentially interpolates the wavelet coefficients and with a desirable property,

$$
\begin{aligned}
& \sum_{k=1}^{N} g_{k}(t)+\mathrm{s}_{\mathrm{gN}}(\mathrm{t})=\mathrm{g}(\mathrm{t}) \\
& \sum_{k=1}^{N} h_{k}(t)+\mathrm{s}_{\mathrm{hN}}(\mathrm{t})=\mathrm{h}(\mathrm{t})
\end{aligned}
$$

where $\mathrm{S}_{\mathrm{gN}}(t)$ and $\mathrm{s}_{\mathrm{hN}}(t)$ are the last smoothing coefficients. An illustration of the wavelet decomposition algorithm is shown in Figure 2. The wavelet we have 
used is the Vetterli's 22-coefficient wavelet. Finally, we note that when a signal is composed of two or more distinct signals or modes, the method proposed here has the advantage that it will find the correct scaling parameter $\eta_{\text {i }}$ for each time-scale, independent of the other time-scales.

\section{SATURATION ESTIMATION WITH SENSOR MOTION}

We now describe a neural network that combines the signal ranks and the amplitude ratio parameters to obtain short-time saturation estimates, and how the sequence of saturation estimates from the neural network is smoothed with filter to obtain a final saturation estimate. The overall estimation algorithm has proven to be useful for analysis of motion corrupted data.

As shown in Figure 3, a backpropagation neural network is used to combine the type of motion with the amplitude ratio parameters of the different scales of the signal to produce the final saturation estimate. The network has seven inputs (corresponding to six amplitude ratio parameters and the rank), one output corresponding to the saturation estimate, and a hidden layer with three hidden nodes. The output of this network is then smoothed or averaged over time to obtain the final saturation estimate of the system. This long time average (approximately $15 \mathrm{~s}$ ) helps reduce the variance of short-time estimates based on 3.5 $\mathrm{s}$ signal segments. The results of this algorithm are compared with a standard algorithm in Figure 4. Note that for stationary sensors, performance of this algorithm is quite similar to the standard algorithm.

Performance of the proposed method in the presence of strong motion and sensor noise is summarized by a receiver operating characteristics (ROC) presented in Figure 6. A false alarm is noted when the saturation estimate falls below a certain level due to sensor noise when in fact the true saturation (obtained from another stationary sensor for the same patient and at the same time) is above the threshold of alarm. Similarly, an true alarm occurs when both the estimate and the known saturation fall below the threshold. Hence, the probability of false alarm versus probability of detection can be estimated with many hours of oximetry data. Care needs to be exercised in the analysis such that periods of continuous alarms are not counted more than once. As shown in Figure 6, the algorithm proposed in this paper has a reduced rate of false alarm at the $80 \%$ detection level. This is an important improvement as false alarm is the most disturbing element in the operation of the pulse oximetry. The reduced detection probability observed in the ROC's is a result of the fact the database was lacking in the number of true alarms and resulting in lower detection probabilities.

\section{SUMMARY AND FUTURE RESEARCH}


We developed a motion detection and saturation estimation algorithm which is based on analysis on waveform properties. While conventional algorithms are based on detecting peaks and troughs of the signal are useful for high signal-to-noise ratio data, the proposed algorithm is designed to perform with data corrupted by motion and sensor noise. We compared the performance with a conventional technique and our preliminary conclusion is that our method is superior. The design is based of computing the autocorrelation, cross-correlation, and spectra of the dual channel oximetry data, and then using neural networks for motion characterization. Wavelet decomposition is used in the saturation estimation as a function of the time scale because the transient properties on the oximetry data are optimally preserved in the wavelet coefficients. A possible research area would be to design an optimal wavelet for the pulse oximetry signals.

\section{ACKNOWLEDGMENTS}

We thank Virgil Kohlhepp and Mitch Lee for developing the codes, evaluating the system and for their many insightful technical contributions in this work. We also thank Tom Yorkey for the fruitful technical discussions and for introducing us to the problem of pulse oximetry. This work was done under a CRADA agreement with Nellcor Puritan Bennett. This work was performed under the auspices of the U.S. Department of Energy by Lawrence Livermore National Laboratory under contract No. W-7405-ENG-48.

\section{REFERENCES}

[1] "Solving Problems in Environmental Engineering and Geosciences with Artificial Neural Networks," F. Dowla and L. Rogers, MIT Press, Cambridge, 1995.

[2]. "Pulse Oximetry." J. F. Kelleher, Journal of Clinical Monitoring, vol. 5, pp. $37-62,1989$.

[3] "Pulse Oximetry," J. P Payne and J. W Severinghaus (Eds.), Springer-Verlag, Berlin, 1986.

[4]. "Pulse Oximetry," K. K. Tremper and S. J. Barker, Anesthesiology, vol. 70, pp. 98-108, 1989.

[5]. "Pulse Oximetry: Analysis of theory, technology, and practice." M . W. Wukitsch, M. T. Petterson, D. R. Tobler, and J. A. Pologe. Journal of Clinical Monitoring, vol. 4, pp. 90-301, 1988.

[6]. "Wavelets and Subband Coding," M. Vetterli and J. Kovacevic, Prentice Hall PTR, Englewood Cliffs, 1995. 


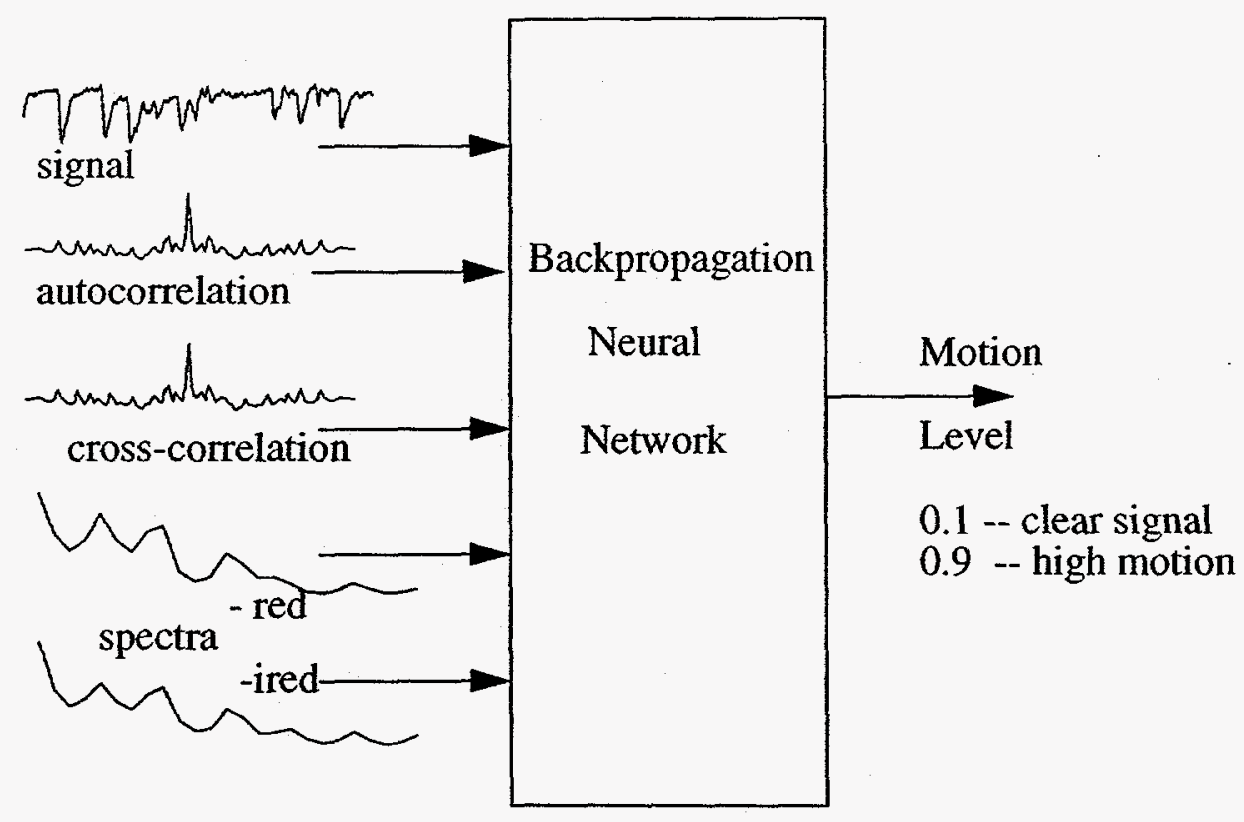

Figure 1: The backpropagation neural network is trained to classify the oximetry signals indicating the presence or absence of motion on a continuous scale from 0.1 (no motion) to 0.9 (high motion). The network uses second-order correlation and spectral data of the red and the infrared signals. 


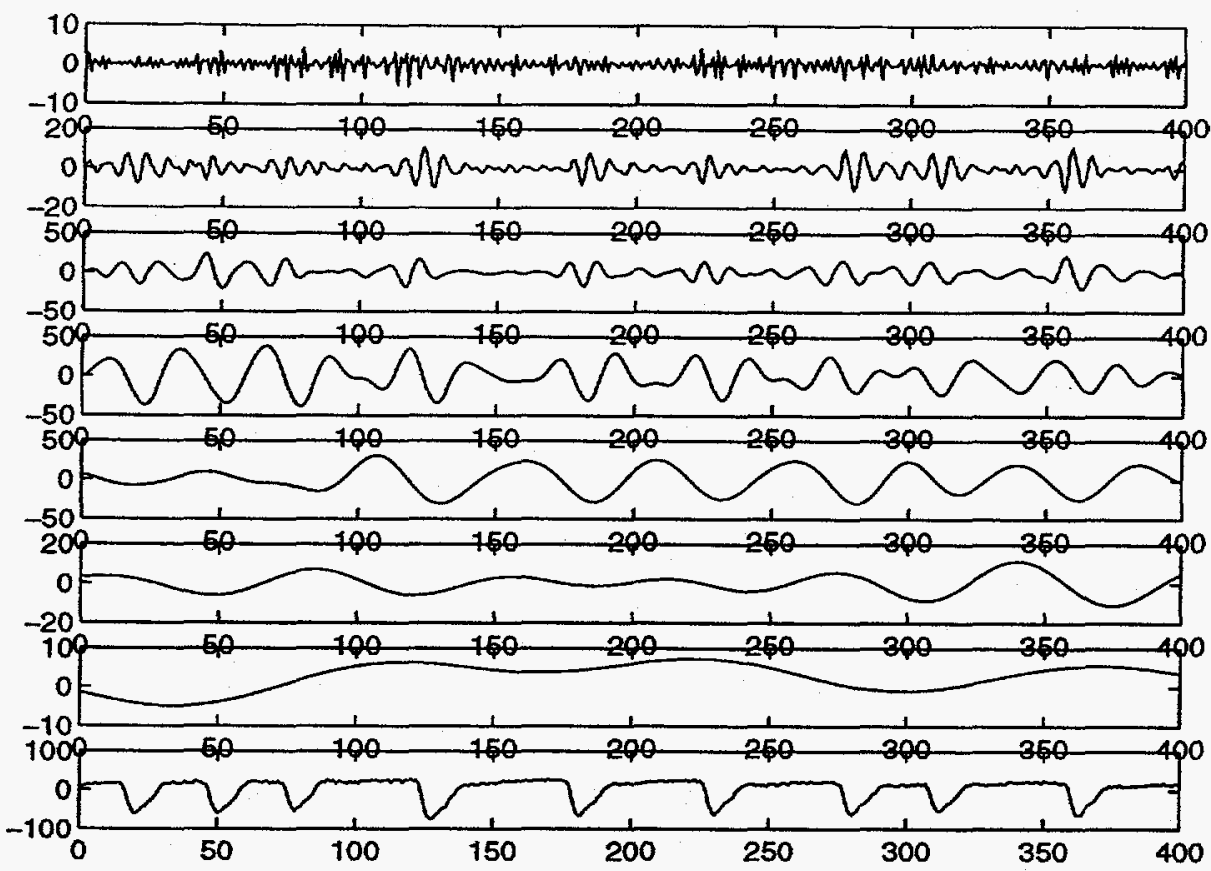

Figure 2: Wavelet multiresolution decomposition of the pulse oximetry signal (bottom) using the Vetterli's 22-point wavelet. The top six signal are the wavelet and the last smoothing coefficients.

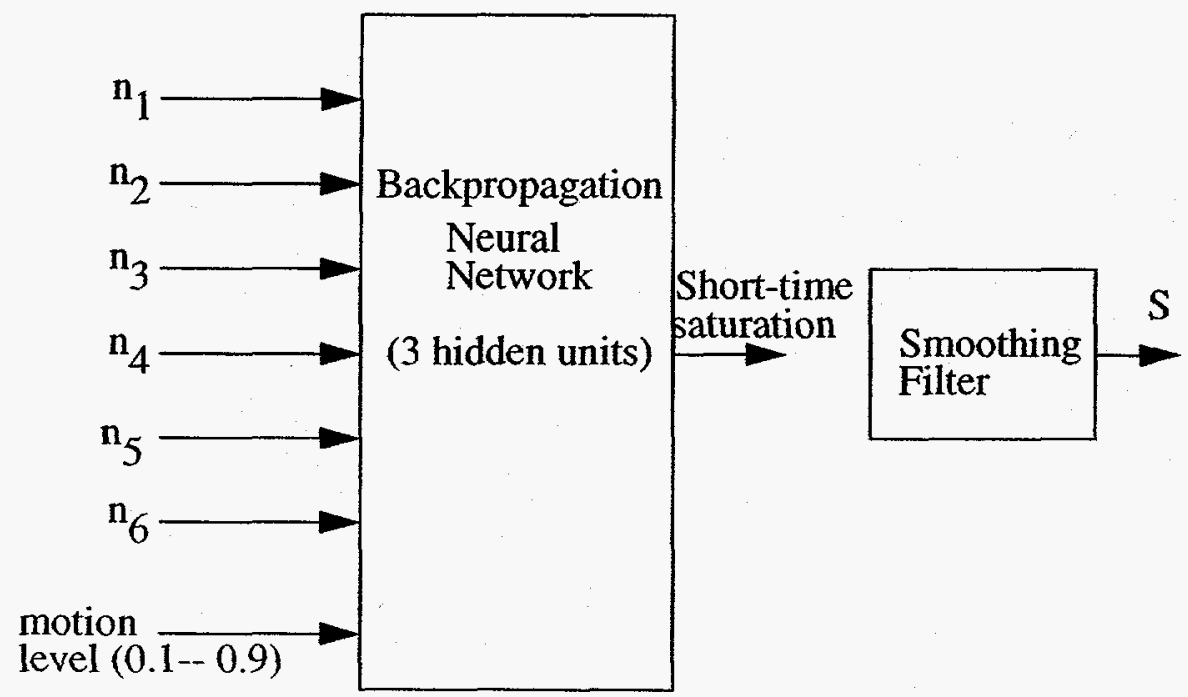

Figure 3: The backpropagation neural network uses the amplitude ratios at different scales and the motion level to estimate the instantaneous saturation level which is smoothed by an smoothing filter. 


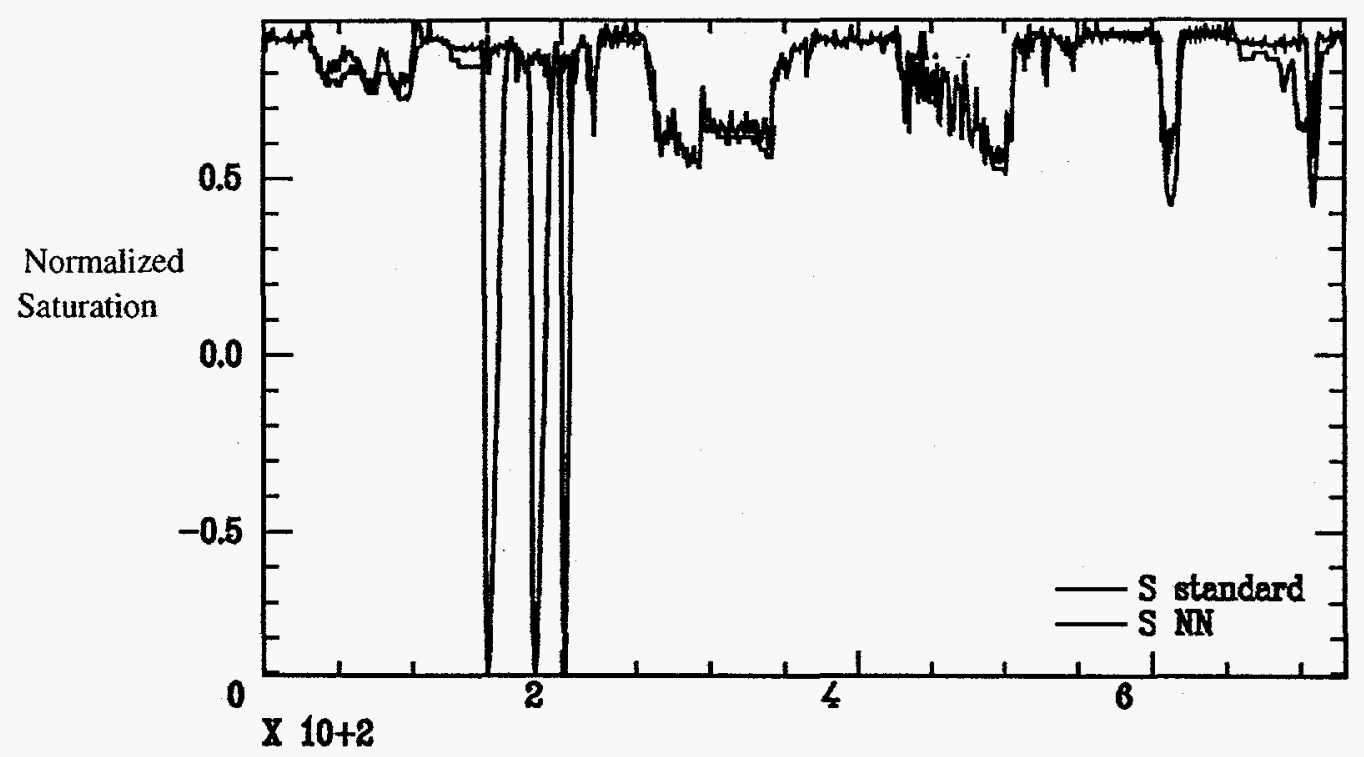

Time (s)

Figure 4: Comparison of the neural network based saturation estimation with a standard algorithm. Unlike the standard algorithm which is unstable during motion, the proposed algorithm tolerates motion much better.

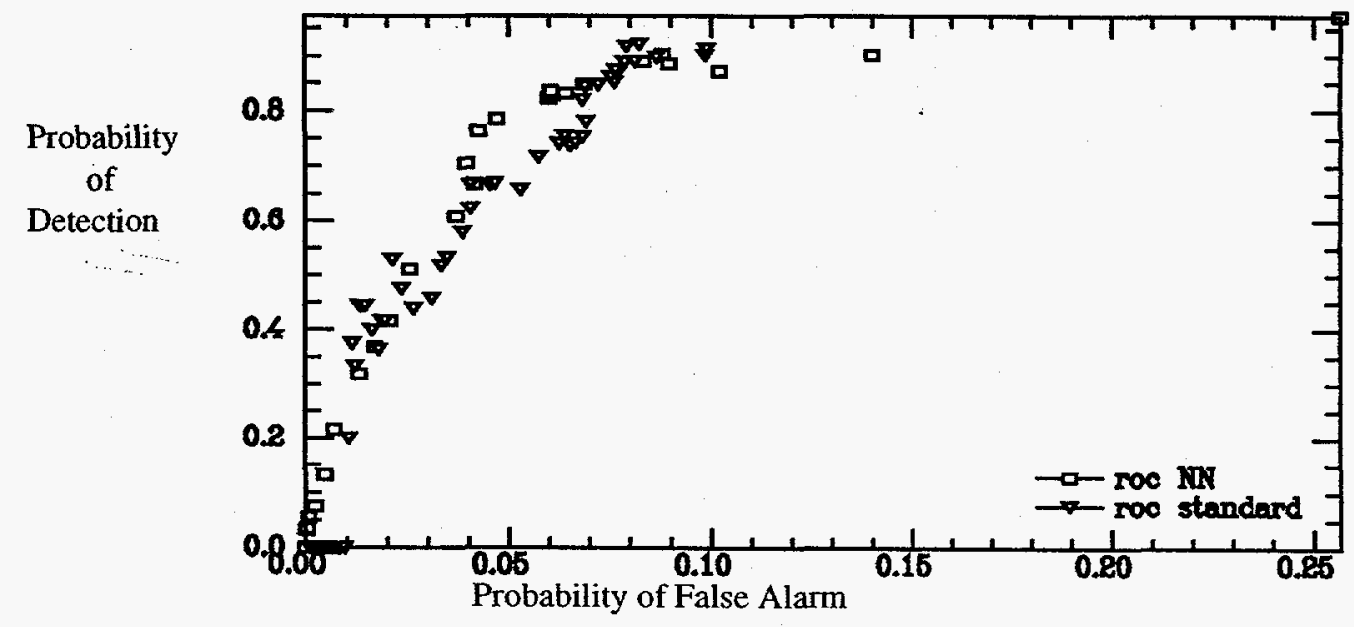

Figure 5: Receiver operating characteristics of the proposed method has less false alarm rate compared to the standard algorithm at the $80 \%$ detection level. 